\title{
CREATING MICROBEAMS AND NANOBEAMS BY CHANNELING IN MICRO- AND NANO-STRUCTURES
}

\author{
V.M. Biryukov, Y.A. Chesnokov, IHEP, Protvino, Russia; S. Bellucci, INFN, Frascati, Italy; V. \\ Guidi, INFN, Ferrara, Italy; W. Scandale, CERN, Geneva, Switzerland
}

\begin{abstract}
A particle beam of very small cross-section is useful in many accelerator applications including biological and medical ones. We show the capability of channeling technique using a micron-sized structure on a surface of a single crystal, or using a nanotube, to produce beam of a cross-section down to 1 square micrometer (or nanometer). The channeled beam can be deflected and well separated in angle and space from the primary and scattered particles. According to our Monte Carlo simulations of channeling and experience with crystal channeling at IHEP (Protvino), emittances down to 0.10.001 nanometer-radian, and flux up to 1 million ions per square micron per second, can be achieved for protons and ions in the range $0.1-3 \mathrm{GeV} / \mathrm{u}$. We discuss the experimental techniques to achieve suitable structures for particle beam channeling.
\end{abstract}

\section{INTRODUCTION}

Bent crystals have efficiently channeled particle beams [1] in the energy range from $3 \mathrm{MeV}$ [2] to $900 \mathrm{GeV}$ [3]. Today, crystals are largely used for extraction of $70-\mathrm{GeV}$ protons at IHEP with efficiency reaching $85 \%$ at intensities well over $10^{12}$ particles/s, steered by silicon crystal as short as $2 \mathrm{~mm}$ [4]. A bent crystal $(5 \mathrm{~mm} \mathrm{Si})$ is installed into the Yellow ring of the Relativistic Heavy Ion Collider where it channels $A u$ ions and polarized protons of $100-250 \mathrm{GeV} / \mathrm{u}$ as a part of the collimation system [5].

Carbon nanotubes are cylindrical molecules made of carbon atoms. Nanotubes can be manufactured of different diameters - from a fraction of a $\mathrm{nm}$ to a few microns, of different lengths - from a micron up to a few millimeters, of different materials - usually carbon but also others [6]. This makes nanotubes a very interesting object for channeling research.

The purpose of the present paper is to look at how the channeling technique could be used to make beams of very small emittance. As a potential application we consider a microbeam facility being developed at BNL [7] where a variety of beams from $\mathrm{Fe}^{+26}$ to protons of 0.1 $3 \mathrm{GeV} / \mathrm{u}$ is needed with the beam size of $\sim 10 \mu \mathrm{m}$ at a target. A traditional approach to creation of a microbeam would be a $\sim 20-\mu \mathrm{m}$-thin wire placed in a circulating beam and a set of micro-collimators cutting out a small part of the scattered-beam phase space [7]. Here the weak points can be a low flux of scattered particles in the direction of extraction line; primary and secondary particles scattered off the collimators may contaminate the microbeam.
If a channeling structure is used instead of wire, it can trap the incident particles and deliver them into a single required direction (i.e. the extraction line) instead of scattering them all the ways around. That may give a large gain in the microbeam flux. The rest of the system may be unchanged: the same set of collimators etc. Further benefit is a low divergence of the channeled beam as set by channeling acceptance; that would reduce the need in collimation down the line, and may reduce the emittance of microbeam. Finally, the channeled beam would have well-defined sharp edges and contain solely primary particles. The open point is how to make a channeling structure as small as about the size of wire, $\sim 0.02 \mathrm{~mm}$, or smaller. Below we suggest two approaches, with crystals and with nanotubes.

\section{CRYSTAL MICRO-BEAM}

The first suggestion is to use a micron-sized structure on a surface of a single crystal; such structures are a welldeveloped technique [8]. The easy way to do it is to take a crystal plate, mask a strip $10 \mu \mathrm{m}$ (or $1 \mu \mathrm{m}$ ) wide on the surface, and etch the surface to the depth of 10 (or 1) $\mu \mathrm{m}$. That leaves a strip of 10 by $10 \mu \mathrm{m}$ (or 1 by $1 \mu \mathrm{m}$ ) on the surface; this strip can channel particles, thus forming a microbeam. In order to separate in the angle and space the beam channeled of the strip from the particles nearby (in the crystal bulk and outside), we suggest having a strip shorter than the substrate plate (Fig.1), and bending the whole structure. That makes a perfect separation downstream.

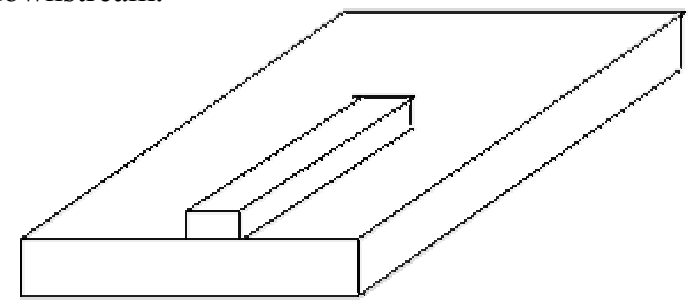

Fig.1 Crystal with a micron-sized strip on the surface.

While the size of the microbeam source is set by the strip size, the divergence in the direction of bending is set by the channeling angle, $(2 \mathrm{Ec} / \mathrm{pv})^{1 / 2}$, where $\mathrm{Ec}$ is the critical transverse energy for channeled particles and $\mathrm{pv}$ is the particle's momentum times velocity per unit charge. E.g., in slightly bent $\mathrm{Si}(100)$ with $\mathrm{Ec} \approx 5 \mathrm{eV}$, the $0.1-3 \mathrm{GeV}$ protons have divergence of $0.05-0.2 \mathrm{mrad}$. For fully stripped ions of $\mathrm{Fe}+26$ in the range of $0.1-1 \mathrm{GeV} / \mathrm{u}$, the divergence is $0.08-0.15 \mathrm{mrad}$. One can pick crystal channels with bigger or smaller angular acceptance. 
With $1-\mu \mathrm{m}$ source, this gives a microbeam emittance of (0.025-0.1) $\pi \mathrm{nm} \cdot$ radian for protons of $0.1-3 \mathrm{GeV}$, and (0.04-0.08) $\pi \mathrm{nm} \cdot$ radian for $\mathrm{Fe}+26$ ions of $0.1-1 \mathrm{GeV} / \mathrm{u}$, in horizontal plane. For comparison, the horizontal emittance expected [7] from the traditional approach is $23 \pi \mathrm{nm} \cdot$ radian at any energy. If realized, the channeling approach would give an improvement by a factor of $\approx 200-1000$ for protons and 300-600 for ions. It can be improved even further by collimation downstream.

In the direction orthogonal to bending, microbeam divergence equals that of the circulating beam. However, the vertical size of microbeam is set by the strip, down to $\sim 1 \mu \mathrm{m}$, while in traditional approach it has to be cut by micro-collimation. Therefore, an improvement $\approx 100$ in vertical emittance can be expected from channeling approach due to small size of the source.

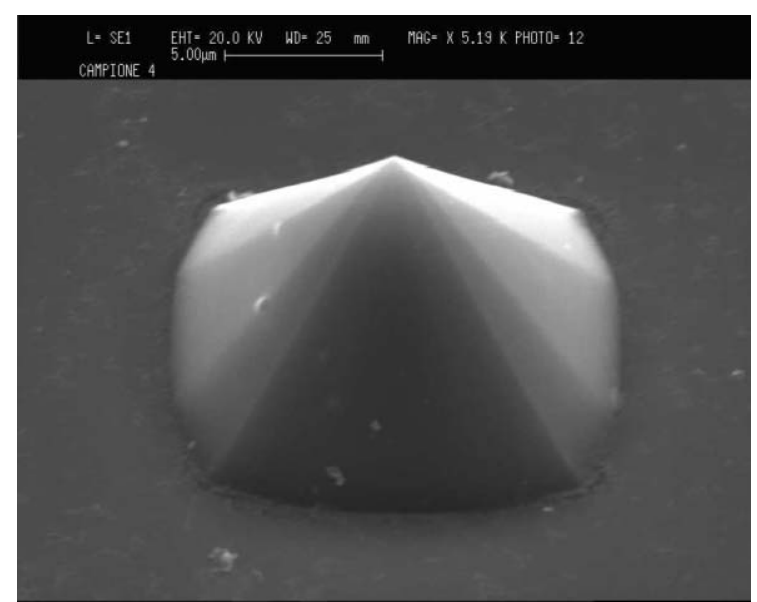

Fig.2 An example of micron-sized structure machined on a Si surface at Ferrara.

Figure 2 shows an example of a micron-sized structure (5-micron pyramid) machined on the surface of Silicon crystal at the Semiconductor group of Ferrara University.

\section{NANO-BEAM}

While crystal channeling is a well-established technique, nanotube channeling is just emerging as a beam instrument [9-13]. Here, beam can be trapped in a single nanotube cylinder of $\approx 1 \mathrm{~nm}$ diameter or in a rope consisting of many nanotubes. The depth $E_{c}$ of the potential well in a carbon nanotube is $\sim 15-60 \mathrm{eV}$ for channeled particles, depending on nanotube configuration [11]. The critical angle for channeling $\theta_{c}=\left(2 E_{c} / p v\right)^{1 / 2}$ is factor of $\sim 1.5-3$ greater than with Si crystal. Provided that nanotubes can efficiently channel and deflect particle beams, they offer an interesting opportunity to make clean beams of potentially very small size, down to 1 square nanometer if needed.

We have developed a Monte Carlo code and done simulations of particle channeling in bent single-wall nanotubes, aimed at finding how useful nanotubes are for channeling of positively-charged particle beams, what kind of nanotubes are efficient for this job, and how nanotubes compare with crystals in this regard [12].

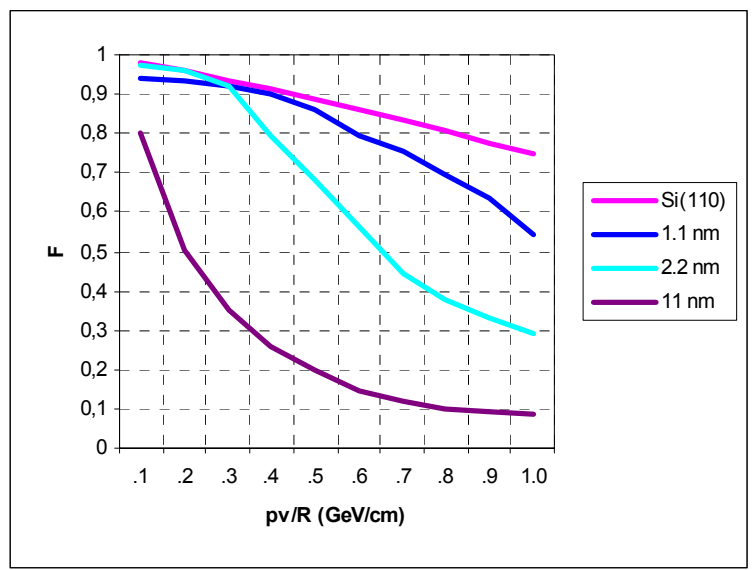

Fig.3 Channeling efficiency vs nanotube curvature $p v / R$ for tubes of different diameter and for $\operatorname{Si}(110)$ crystal.

Figure 3 shows how the number of $1 \mathrm{GeV}$ protons channeled through 50- $\mu \mathrm{m}$-long nanotube depends on nanotube curvature $p v / R$ for tubes of different diameters. For comparison, also shown is the same function for Si(110) crystal. The channel length of $50 \mu \mathrm{m}$, with bending of $1 \mathrm{GeV} / \mathrm{cm}$, gives the $1 \mathrm{GeV}$ particles a deflection of $5 \mathrm{mrad}$ - sufficient for many accelerator applications like extraction [1,3-5]. Nanotube as narrow as $1 \mathrm{~nm}$ is comparable to silicon crystal in beam bending.

For the simulations of nanotube channeling of $\mathrm{Fe}^{+26}$ ions and protons of $0.1-3 \mathrm{GeV} / \mathrm{u}$, we use the tubes of 1.1$\mathrm{nm}$ diameter, typical for easily manufactured carbon nanotubes. We take the curvature radius of $2 \mathrm{~cm}$; then the beam energy range to be studied does nearly correspond to the $p v / R$ range studied in Fig.3. We choose the nanotube bending angle of $5 \mathrm{mrad}$. Figure 4 shows two examples of the angular distribution of protons downstream of the bent nanotube, shown in the direction of bending. Similarly to pictures of bent crystal channeling, there is clear separation of channeled and nonchanneled peaks, with few particles lost (dechanneled along the tube) between themOverall, the transmission of particles by the tube is reasonably good on both ends of the energy range. The intermediate energies fall between the two cases shown.

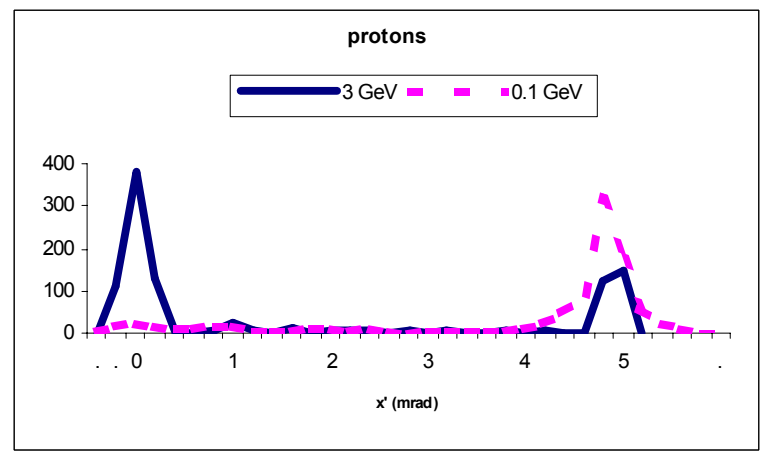

Fig.4 Angular distribution of protons downstream of a bent nanotube, shown for two energies.

The case of $\mathrm{Fe}^{+26}$ ions is shown in Figure 5, again for both ends of the energy range of interest, 0.1 to $1.0 \mathrm{GeV}$ 
per nucleon. Similar picture can be seen, as with protons. Overall, for the similar ratio of beam momentum per unit charge, the angular distributions of $\mathrm{Fe}$ ions and protons are similar. The transmission efficiency is reasonably good for all particle species. The same nanotube deflector could be used in each case, throughout the range of energies and particle species.

For $0.1-1 \mathrm{GeV} / \mathrm{u}$ ions of $\mathrm{Fe}^{+26}$, the divergence of the channeled beam in a nanotube of arbitrary helicity like $(11,9)$ is $0.24-0.77 \mathrm{mrad}$. The size of the source could be quite small. A typical nano-rope (consisting of 100-1000 nanotubes) would be a source that gives an emittance of the nano-beam of the order of $0.001 \pi \mathrm{nm} \cdot$ radian both horizontally and vertically, factor of 10000 down from the figure potentially achievable with a "traditional amorphous" source.

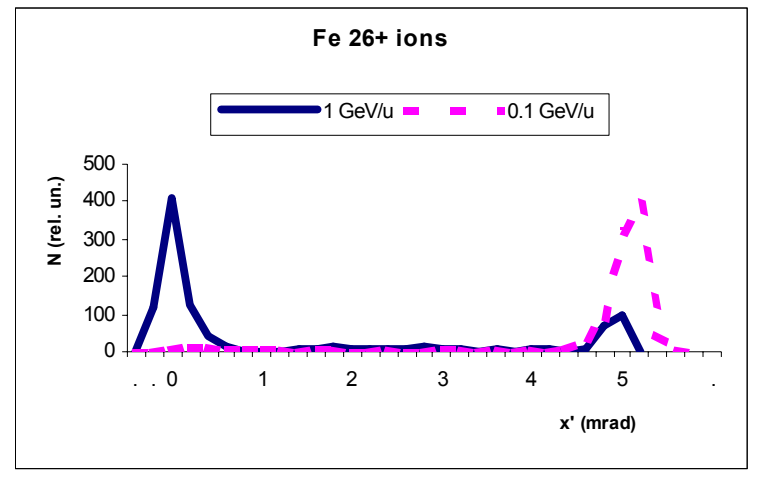

Fig.5 Angular distribution of $\mathrm{Fe}^{+26}$ ions downstream of a bent nanotube, shown for two energies.

\section{INTENSITY OF MICROBEAM}

With small emittance, microbeam intensity is also small. However, the applications like a microbeam facility [7] require quite small intensities, down to 1-1000 particles/s. With some $10^{9}-10^{11}$ particles stored in the AGS ring, this gives enough room for constructing beams of very small emittances discussed above.

Let us take the example of AGS to estimate the achievable intensity of channeled microbeam. The beam circulating in the AGS ring has the size about $\pm 5 \mathrm{~mm}$ before the extraction septum. The beam store is typically $10^{9}$ ions or $10^{11}$ protons. An area of $1 \mu \mathrm{m}^{2}$ would be hit by $\sim 10$ ions (or 1000 protons) in the time of a single turn in the ring $(\sim 1 \mu \mathrm{s}$ at $1 \mathrm{GeV}$ per nucleon $)$; the hit rate is then $\sim 10^{7}$ ions/s per $1 \mu \mathrm{m}^{2}$.

The divergence of particles incident at crystal in periphery of the circulating beam, after crossing a stripping foil, is expected to be several times bigger than channeling acceptance. For particles trapped by a crystal or nanotube, the transmission factor would be 10 to $100 \%$ (e.g., Figs. 4-5) if channeled particles are bent a few mrad.
Microbeam intensity of $10^{5}-10^{7}$ ions/s appears even far greater than needed (though it is easily reduced by moving the crystal away from the core of the beam or misaligning it). One can put the question differently: how much a crystal can survive? The IHEP experience shows that crystals can channel up to $\sim 3 \cdot 10^{12}$ particles/s per cross-section of $0.5 \times 5 \mathrm{~mm}^{2}$ without cooling measures. This corresponds to $10^{6} /\left(\mathrm{s} \cdot \mu \mathrm{m}^{2}\right)=1 /\left(\mathrm{s} \cdot \mathrm{nm}^{2}\right)$. So, a microcrystal structure can channel much more particles than needed, and even a nano-rope could do the job.

A lifetime of $\sim 5 \cdot 10^{20}$ proton irradiation per $\mathrm{cm}^{2}$ as measured [14] for channeling crystal corresponds to $5 \cdot 10^{12} / \mu^{2}$; this means over 100 years of operation of 1 $\mu \mathrm{m}^{2}$ crystal with channeling of $\sim 1000$ protons/s, or one year for $(20-\mathrm{nm})^{2}$ nano-rope operating at 100 protons/s.

\section{ACKNOWLEDGEMENTS}

The authors thank K.A. Brown for discussions and R.P. Fliller for comments. This work was partially supported by INFN - Gruppo V, as NANO experiment, and by INTAS-CERN Grant No. 132-2000.

\section{REFERENCES}

[1] V.M. Biryukov, Yu.A. Chesnokov and V.I. Kotov, "Crystal Channeling and its Application at High Energy Accelerators" (Springer, Berlin, 1997). See also http://crystalbeam.narod.ru

[2] M.B.H. Breese, Nucl. Instr. and Meth. B 132, 540 (1997)

[3] R.A.Carrigan, Jr., et al. Phys. Rev. ST $A B$ 1, 022801 (1998). R.A.Carrigan, Jr., et al. Phys. Rev. ST AB 5, 043501 (2002)

[4] A.G. Afonin, et al. Phys. Rev. Lett. 87, 094802 (2001)

[5] R.P. Fliller III, et al., EPAC 2002 Proceedings (Paris), p.200; these Proceedings.

[6] T.W. Ebbesen, Phys. Today, 49, 26 (1996). Z.Y. Wu, et al., Appl. Phys. Lett. 80, 2973 (2002)

[7] K.A. Brown, et al., EPAC 2002 Proceedings (Paris), p.554; these Proceedings.

[8] P. Kleimann, J. Linnros, R. Juhasz. Appl. Phys. Lett. 79, 1727 (2001)

[9] V.V.Klimov and V.S.Letokhov, Phys. Lett. A 222, 424 (1996)

[10] L.G.Gevorgian, K.A.Ispirian, R.K.Ispirian. JETP Lett. 66, 322 (1997)

[11] N.K. Zhevago and V.I. Glebov, Phys. Lett. A 250, 360 (1998)

[12] V.M. Biryukov and S. Bellucci, Phys. Lett. B 542, 111 (2002)

[13] S. Bellucci, et al., Nucl. Instr. and Meth. B 202, (2003); [ArXiv:physics/0208081]

[14] Baurichter et al., Nucl. Instrum. Meth. B 164165, 27 (2000) 